\title{
Decoupling Zeros of Positive Discrete-Time Linear Systems*
}

\author{
Tadeusz Kaczorek \\ Faculty of Electrical Engineering, Bialystok University of Technology, Bialystok, Poland \\ E-mail:kaczorek@isep.pw.edu.pl \\ Received July 13, 2010; revised August 16, 2010; accepted August 20, 2010
}

\begin{abstract}
The notions of decoupling zeros of positive discrete-time linear systems are introduced. The relationships between the decoupling zeros of standard and positive discrete-time linear systems are analyzed. It is shown that: 1) if the positive system has decoupling zeros then the corresponding standard system has also decoupling zeros, 2) the positive system may not have decoupling zeros when the corresponding standard system has decoupling zeros, 3) the positive and standard systems have the same decoupling zeros if the rank of reachability (observability) matrix is equal to the number of linearly independent monomial columns (rows) and some additional assumptions are satisfied.
\end{abstract}

Keywords: Input-Decoupling Zeros, Output-Decoupling Zeros, Input-Output Decoupling Zeros, Positive, Discrete-Time, Linear, System

\section{Introduction}

In positive systems inputs, state variables and outputs take only non-negative values. Examples of positive systems are industrial processes involving chemical reactors, heat exchangers and distillation columns, storage systems, compartmental systems, water and atmospheric pollution models. A variety of models having positive linear behavior can be found in engineering, management science, economics, social sciences, biology and medicine, etc. An overview of state of the art in positive linear theory is given in the monographs [1,2].

The notions of controllability and observability and the decomposition of linear systems have been introduced by Kalman [3,4]. Those notions are the basic concepts of the modern control theory [5-9]. They have been also extended to positive linear systems $[1,2]$.

The reachability and controllability to zero of standard and positive fractional discrete-time linear systems have been investigated in [10]. The decomposition of positive discrete-time linear systems has been addressed in [11]. The notion of decoupling zeros of standard linear systems have been introduced by Rosebrock $[8,12]$.

In this paper the notions of decoupling zeros will be extended for positive discrete-time linear systems.

The paper is organized as follows. In Section 2 the basic definitions and theorems concerning reachability and

*This work was supported by Ministry of Science and Higher Education in Poland under work No NN514 193933. observability of positive discrete-time linear systems are recalled. The decomposition of the pair $(\mathrm{A}, \mathrm{B})$ and $(\mathrm{A}, \mathrm{C})$ of positive linear system is addressed in Section 3. The main result of the paper is given in Section 4 where the definitions of the decoupling-zeros are proposed and the relationships between decoupling zeros of standard and positive discrete-time linear systems are discussed. Concluding remarks are given in Section 5.

\section{Preliminaries}

The set of $n \times m$ real matrices will be denoted by $\mathfrak{R}^{n \times m}$ and $\mathfrak{R}^{n}:=\mathfrak{R}^{n \times 1}$. The set of $m \times n$ real matrices with nonnegative entries will be denoted by $\mathfrak{R}_{+}^{m \times n}$ and $\mathfrak{R}_{+}^{n}:=\mathfrak{R}_{+}^{n \times 1}$. The set of nonnegative integers will be denoted by $Z_{+}$and the $n \times n$ identity matrix by $I_{n}$.

Consider the linear discrete-time systems

$$
\begin{aligned}
& x_{i+1}=A x_{i}+B u_{i}, \quad i \in Z_{+} \\
& y_{i}=C x_{i}+D u_{i}
\end{aligned}
$$

where $x_{i} \in \mathfrak{R}^{n}, \quad u_{i} \in \mathfrak{R}^{m}, \quad y_{i} \in \mathfrak{R}^{p}$ are the state, input and output vectors and $A \in \mathfrak{R}^{n \times n}, \quad B \in \mathfrak{R}^{n \times m}, C \in \mathfrak{R}^{p \times n}$, $D \in \mathfrak{R}^{p \times m}$.

Definition 2.1. The system (2.1) is called (internally) positive if and only if $x_{i} \in \mathfrak{R}_{+}^{n}$, and $y_{i} \in \mathfrak{R}_{+}^{p}, \quad i \in Z_{+}$ for every $x_{0} \in \mathfrak{R}_{+}^{n}$, and any input sequence $u_{i} \in \mathfrak{R}_{+}^{m}$, $i \in Z_{+}$.

Theorem 2.1. [1,2] The system (2.1) is (internally) positive if and only if 
$A \in \mathfrak{R}_{+}^{n \times n}, \quad B \in \mathfrak{R}_{+}^{n \times m}, C \in \mathfrak{R}_{+}^{p \times n}, \quad D \in \mathfrak{R}_{+}^{p \times m} . \quad$ (2.2)

Definition 2.2. The positive system (2.1) is called reachable in $q$ steps if there exists an input sequence $u_{i} \in \mathfrak{R}_{+}^{m}, \quad i=0,1, \ldots, q-1$ which steers the state of the system from zero $\left(x_{0}=0\right)$ to any given final state $x_{f} \in \mathfrak{R}_{+}^{n}$, i.e., $x_{f}=x_{0}$.

Let $e_{i}, i=1, \ldots, n$ be the $i$ th column of the identity matrix $I_{n}$. A column $a e_{i}$ for $a>0$ is called the monomial column.

Theorem 2.2. [1,2] The positive system (2.1) is reachable in $q$ steps if and only if the reachability matrix

$$
R_{q}=\left[\begin{array}{llll}
B & A B & \ldots & A^{q-1} B
\end{array}\right] \in \mathfrak{R}_{+}^{n \times q m}
$$

contains $n$ linearly independent monomial columns.

Theorem 2.3. [1,2] The positive system (2.1) is reachable in $q$ steps only if the matrix

$$
\left[\begin{array}{ll}
B & A
\end{array}\right]
$$

contains $n$ linearly independent monomial columns.

Definition 2.3. The positive systems (2.1) is called observable in $q$ steps if it is possible to find unique initial state $x_{0} \in \mathfrak{R}_{+}^{n}$ of the system knowing its input sequence $u_{i} \in \mathfrak{R}_{+}^{m}, \quad i=0,1, \ldots, q-1$ and its corresponding output sequence $y_{i} \in \mathfrak{R}_{+}^{p}, \quad i=0,1, \ldots, q-1$.

Theorem 2.4. [1,2] The positive systems (2.1) is observable in $q$ steps if and only if the observability matrix

$$
O_{q}=\left[\begin{array}{c}
C \\
C A \\
\vdots \\
C A^{q-1}
\end{array}\right] \in \mathfrak{R}_{+}^{q p \times n}
$$

contains $n$ linearly independent monomial rows.

Theorem 2.5. [1,2] The positive system (2.1) is observable in $q$ steps only if the matrix

$$
\left[\begin{array}{l}
C \\
A
\end{array}\right]
$$

contains $n$ linearly independent monomial rows.

\section{Decomposition of Positive Pair $(A, B)$ and $(A, C)$ of Positive Linear Systems}

Let the reachability matrix

$$
R_{n}=\left[\begin{array}{llll}
B & A B & \ldots & A^{n-1} B
\end{array}\right] \in \mathfrak{R}_{+}^{n \times m n}
$$

of the positive system (2.1) has $n_{1}<n$ linearly independent monomial columns and let the columns

$$
B_{i_{1}}, B_{i_{2}}, \ldots, B_{i_{k}} \quad(k \leq m)
$$

of the matrix $B \in \mathfrak{R}_{+}^{n \times m}$ be linearly independent monomial columns. We choose from the sequence

$$
A B_{i_{1}}, \ldots, A B_{i_{k}}, A^{2} B_{i_{1}}, \ldots, A^{2} B_{i_{k}}, \ldots, A^{n-1} B_{i_{1}}, \ldots, A^{n-1} B_{i_{k}}
$$

monomial columns which are linearly independent from
(3.2) and previously chosen monomial columns. From those monomial columns we build the monomial matrix

$$
\begin{aligned}
& P=\left[\begin{array}{lllllllllll}
P_{i_{1}} & \ldots & P_{i_{1} d_{1}} & P_{i_{2}} & \ldots & P_{i_{2} d_{2}} & \ldots & P_{i_{k} d_{k}} & P_{n_{1}+1} & \ldots & P_{n}
\end{array}\right] \\
& =\left[\begin{array}{llll}
P_{1} & P_{2} & \ldots & P_{n}
\end{array}\right]
\end{aligned}
$$

where

$$
\begin{aligned}
& P_{i_{1}}=B_{i_{1}}, \ldots, P_{i_{1} d_{1}}=A^{d_{1}-1} B_{i_{1}}, \\
& P_{i_{2}}=B_{i_{2}}, \ldots, P_{i_{2} d_{2}}=A^{d_{2}-1} B_{i_{2}}, \ldots, P_{i_{k} d_{k}}=A^{d_{k}-1} B_{i_{k}}
\end{aligned}
$$

and $d_{i}(i=1, \ldots, k)$ are some natural numbers.

Theorem 3.1. Let the positive system (2.1) be unreachable, the reachability matrix (3.1) have $n_{1}<n$ linearly independent monomial columns and the assumption

$$
P_{k}^{T} A P_{j}=0 \text { for } k=n_{1}+1, \ldots, n ; j=1, \ldots, n_{1}
$$

be satisfied.

Then the pair $(A, B)$ of the system can be reduced by the use of the matrix (3.4) to the form

$$
\begin{aligned}
& \bar{A}=P^{-1} A P=\left[\begin{array}{cc}
\bar{A}_{1} & \bar{A}_{12} \\
0 & \bar{A}_{2}
\end{array}\right], \quad \bar{B}=P^{-1} B=\left[\begin{array}{c}
\bar{B}_{1} \\
0
\end{array}\right], \\
& \bar{A}_{1} \in \mathfrak{R}_{+}^{n_{1} \times n_{1}}, \bar{A}_{2} \in \mathfrak{R}_{+}^{n_{2} \times n_{2}}, \quad\left(n_{2}=n-n_{1}\right) \\
& \bar{A}_{12} \in \mathfrak{R}_{+}^{n_{1} \times n_{2}}, \quad \bar{B}_{1} \in \mathfrak{R}_{+}^{n_{1} \times m}
\end{aligned}
$$

where the pair $\left(\bar{A}_{1}, \bar{B}_{1}\right)$ is reachable and the pair $\left(\bar{A}_{2}, \bar{B}_{2}=0\right)$ is unreachable.

Proof is given in [11].

Theorem 3.2. The transfer matrix

$$
T(z)=C\left[I_{n} z-A\right]^{-1} B+D
$$

of the positive system (2.1) is equal to the transfer matrix

$$
T_{1}(z)=\bar{C}_{1}\left[I_{n_{1}} z-\bar{A}_{1}\right]^{-1} \bar{B}_{1}+D
$$

of its reachable part $\left(\bar{A}_{1}, \bar{B}_{1}, \bar{C}_{1}\right)$, where $C P=\left[\begin{array}{ll}\bar{C}_{1} & \bar{C}_{2}\end{array}\right]$, $\bar{C}_{1} \in \mathfrak{R}_{+}^{p \times n_{1}}$.

Proof is given in [11].

By duality principle [11] we can obtained similar (dual) result for the pair $(A, C)$ of the positive system (2.1).

Let the observability matrix

$$
O_{n}=\left[\begin{array}{c}
C \\
C A \\
\vdots \\
C A^{n-1}
\end{array}\right] \in \mathfrak{R}_{+}^{q n \times n}
$$

has $n_{1}<n$ linearly independent monomial rows.

In a similar way as for the pair $(A, B)$ by the choice of $n_{1}$ linearly independent monomial row for the pair $(A, C)$ we may find the monomial matrix $Q \in \mathfrak{R}_{+}^{n \times n}$ of the form [11]

$$
Q^{T}=\left[\begin{array}{lllllllllll}
Q_{j_{1}}{ }^{T} & \ldots & Q_{j_{1} \bar{d}_{1}}{ }^{T} & Q_{j_{2}}{ }^{T} & \ldots & Q_{j_{2} \bar{d}_{2}}{ }^{T} & \ldots & Q_{j_{l} \bar{d}_{l}}{ }^{T} & Q_{n_{1}+1}{ }^{T} & \ldots & Q_{n}{ }^{T}
\end{array}\right]
$$


where

$$
\begin{aligned}
& Q_{j_{1}}=C_{j_{1}}, \ldots, Q_{j_{1} \bar{d}_{1}}=C_{j_{1}} A^{\bar{d}_{1}-1}, \\
& Q_{j_{2}}=C_{j_{2}}, \ldots, Q_{j_{2} \bar{d}_{2}}=C_{j_{2}} A^{\bar{d}_{2}-1}, \ldots, Q_{j_{l} \bar{d}_{l}}=C_{j_{l}} A^{\bar{d}_{l}-1}
\end{aligned}
$$

and $\bar{d}_{j}(j=1, \ldots, l)$ are some natural numbers.

Theorem 3.3. Let the positive system (2.1) be unobservable, the matrix (3.9) has $n_{1}<n$ linearly independent monomial rows and the assumption

$$
Q_{k} A Q_{j}{ }^{T}=0 \text { for } k=1, \ldots, n_{1} ; j=n_{1}+1, \ldots, n
$$

be satisfied.

Then the pair $(A, C)$ of the system can be reduced by the use of the matrix (3.10) to the form

$$
\begin{aligned}
& \hat{A}=Q A Q^{-1}=\left[\begin{array}{ll}
\hat{A}_{1} & 0 \\
\hat{A}_{21} & \hat{A}_{2}
\end{array}\right], \quad \hat{C}=C Q^{-1}=\left[\begin{array}{ll}
\hat{C}_{1} & 0
\end{array}\right] \\
& \hat{A}_{1} \in \mathfrak{R}_{+}^{n_{1} \times n_{1}}, \quad \hat{A}_{2} \in \mathfrak{R}_{+}^{n_{2} \times n_{2}}, \quad\left(n_{2}=n-n_{1}\right) \\
& \hat{A}_{21} \in \mathfrak{R}_{+}^{n_{2} \times n_{1}}, \quad \hat{C}_{1} \in \mathfrak{R}_{+}^{p \times n_{1}}
\end{aligned}
$$

where the pair $\left(\hat{A}_{1}, \hat{C}_{1}\right)$ is observable and the pair $\left(\hat{A}_{2}, \hat{C}_{2}=0\right)$ is unobservable.

Proof is given in [11].

Theorem 3.4. The transfer matrix (3.7) of the positive system (2.1) is equal to the transfer matrix

$$
T_{1}(z)=\hat{C}_{1}\left[I_{n_{1}} z-\hat{A}_{1}\right]^{-1} \hat{B}_{1}+D
$$

where

$$
Q B=\left[\begin{array}{l}
\hat{B}_{1} \\
\hat{B}_{2}
\end{array}\right], \quad \hat{B}_{1} \in \mathfrak{R}^{n_{1} \times m}, \quad \hat{B}_{2} \in \mathfrak{R}^{n_{2} \times m} .
$$

Proof is given in [11].

Remark 3.1. From Theorem 3.1 and 3.3 it follows that the conditions for decomposition of the pair $(A, B)$ and $(A, C)$ of the positive system (2.1) are much stronger than of the pairs of the standard system.

\section{Decoupling Zeros of the Positive Systems}

It is well-known [5-8] that the input-decupling zeros of standard linear systems are the eigenvalues of the matrix $A_{2}$ of the unreachable (uncontrollable) part of the system. Similarly, the output-decoupling zeros of standard linear systems are the eigenvalues of the matrix of the unreachable and unobservable parts of the system. In a similar way we will defined the decoupling zeros of the positive linear discrete-time systems.

Definition 4.1. Let $\bar{A}_{2} \in \mathfrak{R}_{+}^{\bar{n}_{2} \times \bar{n}_{2}}$ be the matrix of unreachable part of the system (2.1). The zeros $z_{i 1}, z_{i 2}, \ldots, z_{i \bar{n}_{2}}$ of the characteristic polynomial

$$
\operatorname{det}\left[I_{\bar{n}_{2}} z-\bar{A}_{2}\right]=z^{\bar{n}_{2}}+\bar{a}_{\bar{n}_{2}-1} z^{\bar{n}_{2}-1}+\ldots+\bar{a}_{1} z+\bar{a}_{0}
$$

of the matrix $\bar{A}_{2}$ are called the input-decoupling zero of the positive system (2.1).

The list of the input-decoupling zeros will be denoted by $Z_{i}=\left\{z_{i 1}, z_{i 2}, \ldots, z_{i \bar{n}_{2}}\right\}$.

Example 4.1. Consider the positive system (2.1) with the matrices

$$
A=\left[\begin{array}{lll}
1 & 0 & 2 \\
0 & 2 & 0 \\
0 & 0 & 3
\end{array}\right], \quad B=\left[\begin{array}{l}
1 \\
0 \\
0
\end{array}\right]
$$

Note that the pair (4.2) has already the form (3.6) with

$$
\begin{aligned}
& \bar{A}=A=\left[\begin{array}{cc}
\bar{A}_{1} & \bar{A}_{12} \\
0 & \bar{A}_{2}
\end{array}\right]=\left[\begin{array}{lll}
1 & 0 & 2 \\
0 & 2 & 0 \\
0 & 0 & 3
\end{array}\right], \\
& \bar{B}=B=\left[\begin{array}{c}
\bar{B}_{1} \\
0
\end{array}\right]=\left[\begin{array}{l}
1 \\
0 \\
0
\end{array}\right], \quad\left(n_{1}=1, n_{2}=2\right)
\end{aligned}
$$

In this case the characteristic polynomial of the matrix $\bar{A}_{2}=\left[\begin{array}{ll}2 & 0 \\ 0 & 3\end{array}\right]$ has the form

$$
\begin{gathered}
\operatorname{det}\left[I_{2} z-\bar{A}_{2}\right]=\left|\begin{array}{cc}
z-2 & 0 \\
0 & z-3
\end{array}\right|, \\
=(z-2)(z-3)=z^{2}-5 z+6
\end{gathered}
$$

the input-decoupling zeros are equal to $z_{i 1}=2, z_{i 2}=3$ and $Z_{i}=\{2,3\}$.

Definition 4.2. Let $\hat{A}_{2} \in \mathfrak{R}_{+}^{\hat{n}_{2} \times \hat{n}_{2}}$ be the matrix of unobservable part of the system (2.1). The zeros $z_{o 1}, z_{o 2}, \ldots, z_{\overline{o n}_{2}}$ of the characteristic polynomial

$$
\operatorname{det}\left[I_{\hat{n}_{2}} z-\hat{A}_{2}\right]=z^{\hat{n}_{2}}+\hat{a}_{\hat{n}_{2}-1} z^{\hat{n}_{2}-1}+\ldots+\hat{a}_{1} z+\hat{a}_{0}
$$

of the matrix $\hat{A}_{2}$ are called the output-decoupling zero of the positive system (2.1).

The list of the output-decoupling zeros will be denoted by $Z_{o}=\left\{z_{o 1}, z_{o 2}, \ldots, z_{o \hat{n}_{2}}\right\}$.

Example 4.2. Consider the positive system (2.1) with the matrices (4.2) and

$$
C=\left[\begin{array}{lll}
0 & 1 & 0
\end{array}\right], \quad D=[0] .
$$

The observability matrix

$$
O_{3}=\left[\begin{array}{c}
C \\
C A \\
C A^{2}
\end{array}\right]=\left[\begin{array}{lll}
0 & 1 & 0 \\
0 & 2 & 0 \\
0 & 4 & 0
\end{array}\right]
$$

has only one monomial row $Q_{1}=\left[\begin{array}{lll}0 & 1 & 0\end{array}\right]$. In this case the monomial matrix (3.10) has the form

$$
Q=\left[\begin{array}{l}
Q_{1} \\
Q_{2} \\
Q_{3}
\end{array}\right]=\left[\begin{array}{lll}
0 & 1 & 0 \\
1 & 0 & 0 \\
0 & 0 & 1
\end{array}\right]
$$


and the assumption (3.11) is satisfied since

$$
Q_{1} A\left[Q_{2}{ }^{T} \quad Q_{3}^{T}\right]=\left[\begin{array}{lll}
0 & 1 & 0
\end{array}\right]\left[\begin{array}{lll}
1 & 0 & 2 \\
0 & 2 & 0 \\
0 & 0 & 3
\end{array}\right]\left[\begin{array}{ll}
1 & 0 \\
0 & 0 \\
0 & 1
\end{array}\right]=\left[\begin{array}{ll}
0 & 0
\end{array}\right]
$$

Using (3.12) and (4.8) we obtain

$$
\begin{aligned}
\hat{A} & =Q A Q^{-1}=\left[\begin{array}{lll}
0 & 1 & 0 \\
1 & 0 & 0 \\
0 & 0 & 1
\end{array}\right]\left[\begin{array}{lll}
1 & 0 & 2 \\
0 & 2 & 0 \\
0 & 0 & 3
\end{array}\right]\left[\begin{array}{lll}
0 & 1 & 0 \\
1 & 0 & 0 \\
0 & 0 & 1
\end{array}\right] \\
& =\left[\begin{array}{lll}
2 & 0 & 0 \\
0 & 1 & 2 \\
0 & 0 & 3
\end{array}\right]=\left[\begin{array}{ll}
\hat{A}_{1} & 0 \\
\hat{A}_{21} & \hat{A}_{2}
\end{array}\right], \quad\left(n_{1}=1, n_{2}=2\right) \\
\hat{C} & =C Q^{-1}=\left[\begin{array}{ll}
\hat{C}_{1} & 0
\end{array}\right]=\left[\begin{array}{lll}
1 & 0 & 0
\end{array}\right]
\end{aligned}
$$

Characteristic polynomial of the matrix $\hat{A}_{2}=\left[\begin{array}{ll}1 & 2 \\ 0 & 3\end{array}\right]$ has the form

$$
\operatorname{det}\left[I_{2} z-\hat{A}_{2}\right]=\left|\begin{array}{cc}
z-1 & -2 \\
0 & z-3
\end{array}\right|=(z-1)(z-3)=z^{2}-4 z+3
$$

the output-decoupling zeros are equal to $z_{o 1}=1, z_{o 2}=3$ and $Z_{o}=\{1,3\}$.

Definition 4.3. Zeros $z_{i 0}^{(1)}, z_{i 0}^{(2)}, \ldots, z_{i 0}^{(k)}$ which are simultaneously the input-decoupling zeros and the output-decoupling zeros of the positive system (2.1) are called the input-output decoupling zeros of the positive system, i.e.,

$$
z_{i 0}^{(j)} \in Z_{i} \text { and } z_{i 0}^{(j)} \in Z_{o} \text { for } j=1, \ldots, k ; k \leq \min \left(\bar{n}_{2}, \hat{n}_{2}\right)
$$

The list of input-output decoupling zeros will be denoted by $Z_{i 0}=\left\{z_{i 0}^{(1)}, z_{i 0}^{(2)}, \ldots, z_{i 0}^{(k)}\right\}$.

Example 4.3. Consider the positive system (2.1) with the matrices (4.2) and (4.6). The system has the inputdecoupling $z_{i 1}=2, z_{i 2}=3$ and $Z_{i}=\{2,3\} \quad$ (Example 4.1) and the output-decoupling zero $z_{o 1}=1, z_{o 2}=3$ and $Z_{o}=\{1,3\} \quad$ (Example 4.2). Therefore, by Definition 4.3 the positive system has one input-output decoupling zero $z_{i o}=3, Z_{i o}=\{3\}$. This zero is the eigenvalue of the matrix $A_{12}=[3]$ of the unreachable and unobservable part of the system. Note that the transfer function of the system is zero, i.e.,

$$
\begin{aligned}
& T(s)=C\left[I_{n} z-A\right]^{-1} B+D \\
& =\left[\begin{array}{lll}
0 & 1 & 0
\end{array}\right]\left[\begin{array}{ccc}
z-1 & 0 & -2 \\
0 & z-2 & 0 \\
0 & 0 & z-3
\end{array}\right]^{-1}\left[\begin{array}{l}
1 \\
0 \\
0
\end{array}\right]+[0]=[0]
\end{aligned}
$$

since it represents the reachable and observable part of the system.
Example 4.4. Consider the positive system (2.1) with the matrices

$$
A=\left[\begin{array}{lll}
1 & 0 & 2 \\
0 & 2 & 1 \\
0 & 0 & 3
\end{array}\right], \quad B=\left[\begin{array}{l}
1 \\
0 \\
0
\end{array}\right], \quad C=\left[\begin{array}{lll}
0 & 1 & 0
\end{array}\right], \quad D=[0] .
$$

Note that the matrices $B, C, D$ are the same as in Example 4.1 and 4.2 and the matrix $A$ differs by only one entry $a_{23}=1$.

The pair $(A, B)$ has already the form (3.6) since

$$
\begin{aligned}
& \bar{A}=A=\left[\begin{array}{cc}
\bar{A}_{1} & \bar{A}_{12} \\
0 & \bar{A}_{2}
\end{array}\right]=\left[\begin{array}{lll}
1 & 0 & 2 \\
0 & 2 & 1 \\
0 & 0 & 3
\end{array}\right], \\
& \bar{B}=B=\left[\begin{array}{c}
\bar{B}_{1} \\
0
\end{array}\right]=\left[\begin{array}{l}
1 \\
0 \\
0
\end{array}\right], \quad\left(n_{1}=1, n_{2}=2\right)
\end{aligned}
$$

The observability matrix

$$
O_{3}=\left[\begin{array}{c}
C \\
C A \\
C A^{2}
\end{array}\right]=\left[\begin{array}{lll}
0 & 1 & 0 \\
0 & 2 & 1 \\
0 & 4 & 5
\end{array}\right]
$$

has only one monomial row $Q_{1}=\left[\begin{array}{lll}0 & 1 & 0\end{array}\right]$ and

$$
Q=\left[\begin{array}{lll}
0 & 1 & 0 \\
1 & 0 & 0 \\
0 & 0 & 1
\end{array}\right]
$$

is the same as in Example 4.2. The positive pair $(A, C)$ can not be decomposed because the assumption (3.11) is not satisfied, i.e.,

$$
\begin{aligned}
& Q_{1} A\left[Q_{2}{ }^{T} Q_{3}{ }^{T}\right]=\left[\begin{array}{lll}
0 & 1 & 0
\end{array}\right]\left[\begin{array}{lll}
1 & 0 & 2 \\
0 & 2 & 1 \\
0 & 0 & 3
\end{array}\right]\left[\begin{array}{ll}
1 & 0 \\
0 & 0 \\
0 & 1
\end{array}\right] \\
& \quad=\left[\begin{array}{ll}
0 & 1
\end{array}\right] \neq\left[\begin{array}{ll}
0 & 0
\end{array}\right]
\end{aligned}
$$

Now let us consider the standard system (2.1) with (4.12). In this case the matrix (4.14) has two linearly independent rows and

$$
Q=\left[\begin{array}{lll}
0 & 1 & 0 \\
0 & 2 & 1 \\
1 & 0 & 0
\end{array}\right]
$$

Using (3.12) and (4.17) we obtain

$$
\begin{aligned}
\hat{A} & =Q A Q^{-1}=\left[\begin{array}{lll}
0 & 1 & 0 \\
0 & 2 & 1 \\
1 & 0 & 0
\end{array}\right]\left[\begin{array}{lll}
1 & 0 & 2 \\
0 & 2 & 1 \\
0 & 0 & 3
\end{array}\right]\left[\begin{array}{ccc}
0 & 0 & 1 \\
1 & 0 & 0 \\
-2 & 1 & 0
\end{array}\right] \\
& =\left[\begin{array}{ccc}
0 & 1 & 0 \\
-6 & 5 & 0 \\
-4 & 2 & 1
\end{array}\right]=\left[\begin{array}{ll}
\hat{A}_{1} & 0 \\
\hat{A}_{21} & \hat{A}_{2}
\end{array}\right]
\end{aligned}
$$


and

$$
\hat{C}=C Q^{-1}=\left[\begin{array}{ll}
\hat{C}_{1} & 0
\end{array}\right]=\left[\begin{array}{lll}
1 & 0 & 0
\end{array}\right], \quad\left(n_{2}=n-n_{1}\right)
$$

The matrix $\hat{A}_{2}=[1]$ of the unobservable part of the standard system has one eigenvalue which is equal to the output-decoupling zero $z_{o 1}=1$. Note that the standard system has two input-decoupling zeros $z_{i 1}=2, z_{i 2}=3$ and has no input-output decoupling zeros.

The transfer function of the positive and standard system is equal to zero.

Consider the positive pair

$$
A=\left[\begin{array}{ccccc}
0 & 1 & 0 & \ldots & 0 \\
0 & 0 & 1 & \ldots & 0 \\
\vdots & \vdots & \vdots & \ddots & \vdots \\
0 & 0 & 0 & \ldots & 1 \\
a_{0} & a_{1} & a_{2} & \ldots & a_{n-1}
\end{array}\right] \in \mathfrak{R}_{+}^{n \times n}, \quad B=\left[\begin{array}{c}
0 \\
1 \\
0 \\
\vdots \\
0
\end{array}\right] \in \mathfrak{R}_{+}^{n}
$$

with $a_{0}=a_{1}=0$.

The reachability matrix of the pair (4.19)

$$
R_{n}=\left[\begin{array}{llll}
B & A B & \ldots & A^{n-1} B
\end{array}\right]=\left[\begin{array}{ccccc}
0 & 1 & 0 & \ldots & 0 \\
1 & 0 & 0 & \ldots & 0 \\
0 & 0 & 0 & \ldots & 0 \\
\vdots & \vdots & \vdots & \ldots & \vdots \\
0 & 0 & 0 & \ldots & 0
\end{array}\right] \in \mathfrak{R}_{+}^{n \times n}
$$

has rank equal to two and two linearly independent monomial columns.

In this case the monomial matrix (3.4) has the form

$$
P=\left[\begin{array}{lll}
P_{1} & \ldots & P_{n}
\end{array}\right]=\left[\begin{array}{ccccc}
0 & 1 & 0 & \ldots & 0 \\
1 & 0 & 0 & \ldots & 0 \\
0 & 0 & 1 & \ldots & 0 \\
\vdots & \vdots & \vdots & \ddots & \vdots \\
0 & 0 & 0 & \ldots & 1
\end{array}\right] \in \mathfrak{R}_{+}^{n \times n}
$$

and the assumption (3.5) is satisfied since

$$
A P_{2}=\left[\begin{array}{lll}
0 & \ldots & 0
\end{array}\right]^{T} \text { and } P_{k}^{T} A P_{2}=0 \text { for } k=3, \ldots, n \text {. }
$$

Using (3.6) and (4.21) we obtain

$$
\begin{aligned}
& \bar{A}=P^{-1} A P \\
& {\left[\begin{array}{ccccc}
0 & 1 & 0 & \ldots & 0 \\
1 & 0 & 0 & \ldots & 0 \\
0 & 0 & 1 & \ldots & 0 \\
\vdots & \vdots & \vdots & \ddots & \vdots \\
0 & 0 & 0 & \ldots & 1
\end{array}\right]=\left[\begin{array}{cc}
\bar{A}_{1} & \bar{A}_{12} \\
0 & \bar{A}_{2}
\end{array}\right],}
\end{aligned}
$$$$
=\left[\begin{array}{ccccc}
0 & 1 & 0 & \ldots & 0 \\
1 & 0 & 0 & \ldots & 0 \\
0 & 0 & 1 & \ldots & 0 \\
\vdots & \vdots & \vdots & \ddots & \vdots \\
0 & 0 & 0 & \ldots & 1
\end{array}\right]\left[\begin{array}{ccccc}
0 & 1 & 0 & \ldots & 0 \\
0 & 0 & 1 & \ldots & 0 \\
\vdots & \vdots & \vdots & \ddots & \vdots \\
0 & 0 & 0 & \ldots & 1 \\
a_{0} & a_{1} & a_{2} & \ldots & a_{n-1}
\end{array}\right]
$$

$$
\begin{aligned}
\bar{A}_{1} & =\left[\begin{array}{cc}
0 & 0 \\
1 & 0
\end{array}\right] \in \mathfrak{R}_{+}^{2 \times 2}, \bar{A}_{12}=\left[\begin{array}{cccc}
1 & 0 & \ldots & 0 \\
0 & 0 & \ldots & 0
\end{array}\right] \in \mathfrak{R}_{+}^{2 \times(n-2)}, \\
\bar{A}_{2} & =\left[\begin{array}{ccccc}
0 & 1 & 0 & \ldots & 0 \\
0 & 0 & 1 & \ldots & 0 \\
\vdots & \vdots & \vdots & \ddots & \vdots \\
0 & 0 & 0 & \ldots & 1 \\
a_{2} & a_{3} & a_{4} & \ldots & a_{n-1}
\end{array}\right] \in \mathfrak{R}_{+}^{(n-2) \times(n-2)}
\end{aligned}
$$

and

$$
\bar{B}=P^{-1} B=\left[\begin{array}{ccccc}
0 & 1 & 0 & \ldots & 0 \\
1 & 0 & 0 & \ldots & 0 \\
0 & 0 & 1 & \ldots & 0 \\
\vdots & \vdots & \vdots & \ddots & \vdots \\
0 & 0 & 0 & \ldots & 1
\end{array}\right]\left[\begin{array}{c}
0 \\
1 \\
0 \\
\vdots \\
0
\end{array}\right]=\left[\begin{array}{c}
\bar{B}_{1} \\
0
\end{array}\right], \bar{B}_{1}=\left[\begin{array}{c}
1 \\
0
\end{array}\right] \in \mathfrak{R}_{+}^{2}
$$

Theorem 4.1. If the rank of the reachability matrix (4.20) is equal to the number of linearly independent monomial columns then the input-decoupling zeros of the standard and positive system with (4.19) are the same and they are the eigenvalues of the matrix $\bar{A}_{2}$. The state vector $x_{i}$ of the system is independent of the input-decoupling zeros for any input $u_{i}$ and zero initial conditions $\left(x_{0}=0\right)$.

Proof. By Definition 4.1 the input-decoupling zeros are the eigenvalues of the matrix $\bar{A}_{2}$ and they are the same for standard and positive system since the similarity transformation matrix $P$ has in both cases the same form (4.21). If the initial conditions are zero then the zet transformation of $x_{i}$ is given by

$$
\begin{gathered}
X(z)=P^{-1} \bar{X}(z)=P^{-1}[I z-\bar{A}]^{-1} \bar{B} U(z) \\
=P^{-1}\left[\begin{array}{cc}
I z-\bar{A}_{1} & -\bar{A}_{12} \\
0 & I z-\bar{A}_{2}
\end{array}\right]^{-1}\left[\begin{array}{c}
\bar{B}_{1} \\
0
\end{array}\right] U(s) \\
=P^{-1}\left[\begin{array}{c}
{\left[I z-\bar{A}_{1}\right]^{-1} \bar{B}_{1}} \\
0
\end{array}\right] U(z)=\left[\begin{array}{c}
1 \\
z^{-1} \\
0 \\
\vdots \\
0
\end{array}\right] U(z)
\end{gathered}
$$

where $U(z)$ is the zet transform of $u_{i}$.

Dual result we obtain for the positive pair

$$
\begin{aligned}
A & =\left[\begin{array}{ccccc}
0 & 0 & \ldots & 0 & a_{0} \\
1 & 0 & \ldots & 0 & a_{1} \\
0 & 1 & \ldots & 0 & a_{2} \\
\vdots & \vdots & \ddots & \vdots & \vdots \\
0 & 0 & \ldots & 1 & a_{n-1}
\end{array}\right] \in \mathfrak{R}_{+}^{n \times n}, \\
C & =\left[\begin{array}{lllll}
0 & 1 & 0 & \ldots & 0
\end{array}\right] \in \mathfrak{R}_{+}^{1 \times n}
\end{aligned}
$$


with $a_{0}=a_{1}=0$.

The observability matrix of the pair (4.24)

$$
O_{n}=\left[\begin{array}{c}
C \\
C A \\
\vdots \\
C A^{n-1}
\end{array}\right]=\left[\begin{array}{ccccc}
0 & 1 & 0 & \ldots & 0 \\
1 & 0 & 0 & \ldots & 0 \\
0 & 0 & 0 & \ldots & 0 \\
\vdots & \vdots & \vdots & \ldots & \vdots \\
0 & 0 & 0 & \ldots & 0
\end{array}\right] \in \mathfrak{R}_{+}^{n \times n}
$$

has rank equal to two and two linearly independent monomial rows.

In this case the monomial matrix (3.10) has the form

$$
P=\left[\begin{array}{c}
Q_{1} \\
\vdots \\
Q_{n}
\end{array}\right]=\left[\begin{array}{ccccc}
0 & 1 & 0 & \ldots & 0 \\
1 & 0 & 0 & \ldots & 0 \\
0 & 0 & 1 & \ldots & 0 \\
\vdots & \vdots & \vdots & \ddots & \vdots \\
0 & 0 & 0 & \ldots & 1
\end{array}\right] \in \mathfrak{R}_{+}^{n \times n}
$$

and the assumption (3.11) is satisfied since $Q_{2} A=\left[\begin{array}{lll}0 & \ldots & 0\end{array}\right]$ and $Q_{2} A Q_{k}=0$ for $k=3, \ldots, n$.

Using (3.12) and (4.25) we obtain

$$
\begin{aligned}
& \hat{A}=Q A Q^{-1} \\
& =\left[\begin{array}{ccccc}
0 & 1 & 0 & \ldots & 0 \\
1 & 0 & 0 & \ldots & 0 \\
0 & 0 & 1 & \ldots & 0 \\
\vdots & \vdots & \vdots & \ddots & \vdots \\
0 & 0 & 0 & \ldots & 1
\end{array}\right]\left[\begin{array}{ccccc}
0 & 0 & \ldots & 0 & a_{0} \\
1 & 0 & \ldots & 0 & a_{1} \\
0 & 1 & \ldots & 0 & a_{2} \\
\vdots & \vdots & \ddots & \vdots & \vdots \\
0 & 0 & \ldots & 1 & a_{n-1}
\end{array}\right] \\
& {\left[\begin{array}{ccccc}
0 & 1 & 0 & \ldots & 0 \\
1 & 0 & 0 & \ldots & 0 \\
0 & 0 & 1 & \ldots & 0 \\
\vdots & \vdots & \vdots & \ddots & \vdots \\
0 & 0 & 0 & \ldots & 1
\end{array}\right]=\left[\begin{array}{ll}
\hat{A}_{1} & 0 \\
\hat{A}_{21} & \hat{A}_{2}
\end{array}\right],} \\
& \hat{A}_{1}=\left[\begin{array}{cc}
0 & 1 \\
0 & 0
\end{array}\right] \in \mathfrak{R}_{+}^{2 \times 2}, \quad \hat{A}_{21}=\left[\begin{array}{cc}
1 & 0 \\
0 & 0 \\
\vdots & \vdots \\
0 & 0
\end{array}\right] \in \mathfrak{R}_{+}^{(n-2) \times 2}, \\
& \hat{A}_{2}=\left[\begin{array}{ccccc}
0 & 0 & \ldots & 0 & a_{2} \\
1 & 0 & \ldots & 0 & a_{3} \\
0 & 1 & \ldots & 0 & a_{4} \\
\vdots & \vdots & \ddots & \vdots & \vdots \\
0 & 0 & \ldots & 1 & a_{n-1}
\end{array}\right] \in \mathfrak{R}_{+}^{(n-2) \times(n-2)}
\end{aligned}
$$

and

$$
\begin{gathered}
\hat{C}=C Q^{-1}=\left[\begin{array}{lllll}
0 & 1 & 0 & \ldots & 0
\end{array}\right]\left[\begin{array}{ccccc}
0 & 1 & 0 & \ldots & 0 \\
1 & 0 & 0 & \ldots & 0 \\
0 & 0 & 1 & \ldots & 0 \\
\vdots & \vdots & \vdots & \ddots & \vdots \\
0 & 0 & 0 & \ldots & 1
\end{array}\right] \\
=\left[\begin{array}{llll}
1 & 0 & \ldots & 0
\end{array}\right]=\left[\begin{array}{ll}
\hat{C}_{1} & 0
\end{array}\right], \quad \hat{C}_{1}=\left[\begin{array}{ll}
1 & 0
\end{array}\right] \in \mathfrak{R}_{+}^{1 \times 2}
\end{gathered}
$$

Theorem 4.2. If the rank of the observability matrix (4.25) is equal to the number of linearly independent monomial rows then the output-decoupling zeros of the standard and positive system with (4.24) are the same and they are the eigenvalues of the matrix $\hat{A}_{2}$. The output $y_{i}$ of the system is independent of the output-decoupling zeros for any input $u_{i}{ }^{\prime}=B u_{i}$ and zero initial conditions $\left(x_{0}=0\right)$.

Proof is similar (dual) to the proof of Theorem 4.1.

Example 4.5. For the positive pair

$$
A=\left[\begin{array}{lll}
0 & 0 & 0 \\
1 & 0 & 0 \\
0 & 1 & a
\end{array}\right], \quad(a>0), C=\left[\begin{array}{lll}
0 & 1 & 0
\end{array}\right]
$$

the matrix (4.26) has the form

$$
Q=\left[\begin{array}{lll}
0 & 1 & 0 \\
1 & 0 & 0 \\
0 & 0 & 1
\end{array}\right] .
$$

Using (3.12) and (4.29) we obtain

$$
\begin{aligned}
& \hat{A}=Q A Q^{-1}=\left[\begin{array}{lll}
0 & 1 & 0 \\
0 & 0 & 0 \\
1 & 0 & a
\end{array}\right]=\left[\begin{array}{ll}
\hat{A}_{1} & 0 \\
\hat{A}_{21} & \hat{A}_{2}
\end{array}\right], \\
& \hat{A}_{1}=\left[\begin{array}{ll}
0 & 1 \\
0 & 0
\end{array}\right], \quad \hat{A}_{21}=\left[\begin{array}{ll}
1 & 0
\end{array}\right], \quad \hat{A}_{2}=[a]
\end{aligned}
$$

and

$$
C=C Q^{-1}=\left[\begin{array}{lll}
1 & 0 & 0
\end{array}\right]=\left[\begin{array}{ll}
\hat{C}_{1} & 0
\end{array}\right], \hat{C}_{1}=\left[\begin{array}{ll}
1 & 0
\end{array}\right]
$$

The pair $\left(\hat{A}_{1}, \hat{C}_{1}\right)$ is observable since

$\left[\begin{array}{c}\hat{C}_{1} \\ \hat{C}_{1} \hat{A}_{1}\end{array}\right]=\left[\begin{array}{cc}1 & 0 \\ 0 & 1\end{array}\right]$ and the positive system has one ouputdecoupling zero $z_{01}=a$.

The zet transform of the output for $x_{o}=0$ and $U^{\prime}(z)=B U(z)$ is given by

$$
\begin{aligned}
& T(s)=C\left[I_{n} z-A\right]^{-1} U^{\prime}(z) \\
& \quad=\hat{C}\left[I_{n} z-\hat{A}\right]^{-1} U^{\prime}(z)=z^{-1} U^{\prime}(z)
\end{aligned}
$$

and it is independent of the output-decoupling zero.

The presented results can be extended to multi-input multi-output discrete-time linear systems as follows.

Theorem 4.3. Let the reachability matrix (3.1) of the positive system (2.1) have rank equal to its $n_{1}<n$ line- 
arly independent monomial columns and the assumption (3.5) be satisfied. Then the input-decoupling zeros of the standard and positive system are the same and they are the eigenvalues of the matrix $\bar{A}_{2}$. The state vector $x_{i}$ of the system is independent of the input-decoupling zeros for any input vector $u_{i}$ and zero initial conditions.

Proof. If the reachability matrix (3.1) of the system (2.1) has rank equal to its $n_{1}$ linearly independent monomial columns and the assumption (3.5) is satisfied then the similarity transformation matrix $P$ has the same form for standard and positive system. In this case the matrix $\bar{A}_{2}$ is the same for standard and positive system. Therefore, the input-decoupling zero for the standard and positive system is the same. The second part of the Theorem can be proved in a similar way as of Theorem 4.1.

Theorem 4.4. Let the observability matrix (3.9) of the positive system (2.1) has rank equal to its $n_{1}<n$ linearly independent monomial rows and the assumption (3.11) be satisfied. Then the output-decoupling zeros of the standard and positive system are the same and they are the eigenvalues of the matrix $\hat{A}_{2}$. The output vector $y_{i}$ of the system is independent of the output-decoupling zeros for any input vector $u_{i}{ }^{\prime}=B u_{i}$ and zero initial conditions.

Remark 4.1. Note that if the positive pair $(A, B)$ can be decomposed then the corresponding standard pair $(A, B)$ can also be decomposed. Therefore, if the positive system (2.1) has input-decoupling zeros then the standard system (2.1) has also input-decoupling zeros.

Similar (dual) remark we have for the pair $(A, C)$ and the output-decoupling zeros.

The following example shows that the positive system (2.1) may not have input-decoupling zeros but the standard system has input-decoupling zeros.

Example 4.6. The reachability matrix for the positive pair

$$
A=\left[\begin{array}{lll}
1 & 2 & 1 \\
0 & 1 & 0 \\
1 & 3 & 1
\end{array}\right], \quad B=\left[\begin{array}{l}
1 \\
0 \\
1
\end{array}\right]
$$

has the form

$$
\left[\begin{array}{lll}
B & A B & A^{2} B
\end{array}\right]=\left[\begin{array}{lll}
1 & 2 & 4 \\
0 & 0 & 0 \\
1 & 2 & 4
\end{array}\right] .
$$

It has no monomial columns and it can not be decomposed (as the positive pair) but it can be decomposed as a standard pair since the rank of the reachability matrix (4.33) is equal to one. The similarity transformation matrix has the form

$$
P=\left[\begin{array}{lll}
1 & 0 & 0 \\
0 & 1 & 0 \\
1 & 0 & 1
\end{array}\right]
$$

and we obtain

$$
\begin{gathered}
\bar{A}=P^{-1} A P=\left[\begin{array}{lll}
2 & 2 & 1 \\
0 & 1 & 0 \\
0 & 1 & 0
\end{array}\right]=\left[\begin{array}{cc}
\bar{A}_{1} & \bar{A}_{12} \\
0 & \bar{A}_{2}
\end{array}\right], \\
\bar{A}_{1}=[2], \bar{A}_{12}=\left[\begin{array}{ll}
2 & 1
\end{array}\right], \bar{A}_{2}=\left[\begin{array}{ll}
1 & 0 \\
1 & 0
\end{array}\right] \\
\bar{B}=P^{-1} B=\left[\begin{array}{l}
1 \\
0 \\
0
\end{array}\right]=\left[\begin{array}{c}
\bar{B}_{1} \\
0
\end{array}\right], \bar{B}_{1}=[1]
\end{gathered}
$$

The matrix $\bar{A}_{2}$ has the eigenvalues $z_{i 1}=1, z_{i 2}=0$. Therefore, the positive system with (4.32) has not input-decoupling zeros but it has input-decoupling zeros $\left(z_{i 1}=1, z_{i 2}=0\right)$ as a standard system.

\section{Concluding Remarks}

The notions of the input-decoupling zero, output-decoupling zero and input-output decoupling zero for positive discrete-time linear systems have been introduced. The necessary and sufficient conditions for the reachability (observability) of positive linear systems are much stronger than the conditions for standard linear systems (Theorem 2.2 and 2.4). The conditions for decomposition of positive system are also much stronger than for the standard systems. Therefore, the conditions for the existence of decoupling zeros of positive systems are more restrictive. It has been shown that: 1) if the positive system has decoupling zeros then the corresponding standard system has also decoupling zeros, 2) the positive system may not have decoupling zeros when the corresponding standard system has decoupling zeros (Example 4.6), 3) the positive and standard system have the same decoupling zeros if the rank of reachability (observability) matrix is equal to the number of linearly independent monomial columns (rows) and the assumption (3.5) ((3.11)) is satisfied (Theorem 4.3 and 4.4).

The considerations have been illustrated by numerical examples. Open problems are extension of these considerations to positive continuous-time linear systems and to positive 2D linear systems.

\section{References}

[1] L. Farina and S. Rinaldi, "Positive Linear Systems, Theory and Applications," Wiley, New York, 2000.

[2] T. Kaczorek, "Positive 1D and 2D Systems," Springer Verlag, London, 2001.

[3] R. E. Kalman, "Mathematical Descriptions of Linear Systems," SIAM Journal on Control, Vol. 1, No. 2, 1963, pp. 152-192.

[4] R. E. Kalman, "On the General Theory of Control Sys- 
tems," Proceedings of the First International Congress on Automatic Control, Butterworth, London, 1960, pp. 481-493.

[5] P. J. Antsaklis and A. N. Michel, "Linear Systems," Birkhauser, Boston, 2006.

[6] T. Kaczorek, "Linear Control Systems," Vol. 1, Wiley, New York, 1993.

[7] T. Kailath, "Linear Systems," Prentice-Hall, Englewood Cliffs, New York, 1980.

[8] H. H. Rosenbrock, "State-Space and Multivariable Theory," Wiley, New York, 1970.

[9] W. A. Wolovich, "Linear Multivariable Systems," Springer-Verlag, New York, 1974.
[10] T. Kaczorek, "Reachability and Controllability to Zero Tests for Standard and Positive Fractional Discrete-Time Systems," Journal Européen des Systèmes Automatisés, Vol. 42, No. 6-8, 2008, pp. 770-781.

[11] T. Kaczorek, "Decomposition of the Pairs (A,B) and (A,C) of the Positive Discrete-Time Linear Systems," Proceedings of TRANSCOMP, Zakopane, 6-9 December 2010.

[12] H. H. Rosenbrock, "Comments on Poles and Zeros of Linear Multivariable Systems: A Survey of the Algebraic Geometric and Complex Variable Theory," International Journal on Control, Vol. 26, No. 1, 1977, pp. 157-161. 